\title{
POINTWISE ESTIMATES FOR HIGHER ORDER CONVEXITY PRESERVING POLYNOMIAL APPROXIMATION
}

\author{
JIA-DING CAO ${ }^{1}$ and HEINZ H. GONSKA ${ }^{2}$
}

(Received 3 March 1993)

Dedicated to Professor Dr W. Meyer-König on the occasion of his 80th birthday

Abstract

\begin{abstract}
DeVore-Gopengauz-type operators have attracted some interest over the recent years. Here we investigate their relationship to shape preservation. We construct certain positive convolution-type operators $H_{n, s, j}$ which leave the cones of $j$-convex functions invariant and give Timan-type inequalities for these. We also consider Boolean sum modifications of the operators $H_{n, s, j}$, show that they basically have the same shape preservation behavior while interpolating at the endpoints of $[-1,1]$, and also satisfy Telyakovskiî- and DeVoreGopengauz-type inequalities involving the first and second order moduli of continuity, respectively. Our results thus generalize related results by Lorentz and Zeller, Shvedov, Beatson, DeVore, Yu and Leviatan.
\end{abstract}

\section{Introduction}

The present note deals with shape preservation by so-called DeVore-Gopengauz-type approximants and thus falls into the general context of approximation with constraints (in connection with pointwise estimates). See, for example, Section 3 of [24] and the excellent survey on the early history of the subject in Section 3.2 of [14] which also contains numerous references.

Certain approximation processes which preserve shape properties of a function, and the parametric curves and surfaces based upon them nowadays are not only widely used in Computer-Aided Design, but also to represent curves, surfaces, and volumes for which the data are not necessarily subject to modification (including physical and physiological data, for example). A well-established tool in these fields is the Bernstein-Bézier technique, which was derived from the classical Bernstein operators. However, the present authors feel that the possibilities provided by approximation

\footnotetext{
${ }^{1}$ Dept of Mathematics, Fudan University, Shanghai, PRC.

${ }^{2}$ Dept of Mathematics, University of Duisburg, D-47048 Duisburg, Germany.

(C) Australian Mathematical Society, 1994, Serial-fee code 0334-2700/94
} 
theory have not yet been fully exploited in the fields mentioned. The present paper aims to bridge the gap between good shape preservation properties and the best possible degree of approximation (not rendered by Bernstein operators). Further details are given below.

To define DeVore-Gopengauz operators we first introduce some notation. Let $\mathbb{N}:=\{1,2, \ldots\}$ be the set of natural numbers, $\mathbb{Z}:=\{0, \pm 1, \pm 2, \ldots\}, C[a, b]$ the real vector space of continuous functions on the closed interval $[a, b]$ and $\Pi_{n}$ be its subspace consisting of all algebraic polynomials of degree $\leq n$. By $\omega_{2}(f, \cdot)$ we denote the second order modulus of smoothness of $f \in C[a, b]$, given for $0 \leq \delta \leq(b-a) / 2$ by

$$
\omega_{2}(f, \delta):=\sup \{|f(x-h)-2 f(x)+f(x+h)|: x, x \pm h \in[a, b], 0 \leq h \leq \delta\} .
$$

DEFINITION 1.1. A sequence of linear operators $L_{n}: C[-1,1] \rightarrow \Pi_{r n+s}, r \in \mathbb{N}$, $s \in \mathbb{Z}$ fixed, is said to be of DeVore-Gopengauz-type, if for all $f \in C[-1,1]$ and all $x \in[-1,1]$ one has

$$
\left|f(x)-L_{n}(f, x)\right| \leq c \cdot \omega_{2}\left(f, \frac{\sqrt{1-x^{2}}}{n}\right)
$$

with a constant $c$ independent of $n, f$ and $x$.

Two remarkable features of DeVore-Gopengauz-type operators are the facts that they reproduce linear function, that is, $L_{n}(f, \cdot)=f$ for all $f \in \Pi_{1}$, and that they interpolate the approximated function of the endpoints of its interval of definition. Both properties are shared by the classical Bernstein operators which have other remarkable features, too.

In order to further motivate our research below we recall the following facts. It is well known that the Bemstein operators $B_{n}$ mapping $C[0,1]$ into $\Pi_{n}$ preserve convexity of all orders, that is, $f^{(j)} \geq 0$ on $[0,1]$ implies $\left[B_{n}(f)\right]^{(j)} \geq 0$ on $[0,1]$ for $j=0,1, \ldots$. It is for this reason (among others) that they have received considerable interest in Approximation Theory and, more recently, also in ComputerAided Geometric Design (see [17] and [20]). However, their rate of approximation is poor in comparison to that of DeVore-Gopengauz-type operators. A result dating from 1964 ([4]) states that

$$
\left|B_{n}(f, x)-f(x)\right| \leq c \omega_{2}(f ; \sqrt{x(1-x) / n}) \text { for all } f \in C[0,1] \text {, all } x \in[0,1] .
$$

Only recently inequalities of this type have again attracted some interest (see, for example, [27] and the references cited there). The saturation theorem by Lorentz [23] and the inverse theorems of Berens and Lorentz [3] show that this is, in a certain 
sense, indeed best possible for Bernstein operators. The following result by Berens and DeVore [2] demonstrates that this slow degree of approximation is a consequence of their convexity, and that they have the best rate of approximation among all operators with the same shape preserving properties.

THEOREM A. Let $\mathbf{L}_{n}$ denote the class of all operators $L_{n}$ mapping $C[0,1]$ into itself with

(i) $L_{n}(f) \in \Pi_{n}$ for all $f \in C[0,1]$,

(ii) $L_{n}(l)=$ l for all $l \in \Pi_{1}$,

(iii) $\left[L_{n}(f)\right]^{(j)} \geq 0$, if $f^{(j)} \geq 0, j=0,1, \ldots, n$.

Then

$$
\frac{x(1-x)}{n}=B_{n}\left[(\cdot-x)^{2}, x\right]=\inf _{L_{n} \in \mathbf{L}_{n}} L_{n}\left[(\cdot-x)^{2}, x\right], \quad 0 \leq x \leq 1 .
$$

The assumption (iii) of Theorem $\mathrm{A}$, that all cones of $j$-convex functions, $j=$ $0,1, \ldots, n$, be invariant under the operators in the class $\mathbf{L}_{n}$, is quite stringent. It is therefore a natural question if this assumption can be loosened somewhat in order to get a better rate of convergence. This is the topic addressed in the present note which can thus be viewed as written in the spirit of the Berens-DeVore paper.

The method to be examined below is quite classical, while still offering capabilities which seem to have been overlooked before. We start off with a short description of this technique.

The traditional method of proving Jackson's theorem for $f, g \in C[a, b]$ uses convolution operators of the form

$$
G(f, x)=(f * g)(x)=\frac{1}{\pi} \int_{-\pi}^{\pi} f(\cos t) g[\cos (\theta-t)] d t, \quad x=\cos \theta .
$$

If $K_{m(n)}$ is an even trigonometric kernel of the form

$$
K_{m(n)}(\nu)=\frac{1}{2}+\sum_{k=1}^{m(n)} \rho_{k, m(n)} \cdot \cos k \nu
$$

then

$$
\begin{aligned}
g_{m(n)}(z) & :=K_{m(n)}(\arccos z) \\
& =\frac{1}{2}+\sum_{k=1}^{m(n)} \rho_{k, m(n)} \cdot T_{k}(z)
\end{aligned}
$$

where $T_{k}$ denotes the $k$-th Čebyšev polynomial, is an element of $\Pi_{m(n)}$. 
Furthermore, $G=G_{m(n)}$ attains the form $(\theta=\arccos x)$

$$
\begin{aligned}
G_{m(n)}(f, x) & =\frac{1}{\pi} \int_{-\pi}^{\pi} f(\cos t) \cdot K_{m(n)}[\arccos (\cos (\theta-t))] d t \\
& =\frac{1}{\pi} \int_{-\pi}^{\pi} f(\cos t) \cdot K_{m(n)}(\arccos x-t) d t \\
& =\frac{1}{\pi} \int_{-\pi}^{\pi} f[\cos (\arccos x+t)] \cdot K_{m(n)}(t) d t,
\end{aligned}
$$

and, for each $f, G_{m(n)}(f, \cdot)$ is also in $\Pi_{m(n)}$.

In previous research an important role has been played by positive kernels $K_{m(n)}$ and in particular by the higher order Jackson kernels (Matsuoka kernels) given as follows (see, for example, [15], [26]).

$$
\text { For } s \in \mathbb{N}, \quad \text { let } K_{s n-s}(v):=c_{n, s}\left(\frac{\sin (n v / 2)}{\sin (v / 2)}\right)^{2 s},
$$

where $c_{n, s}$ is chosen so that $\pi^{-1} \int_{-\pi}^{\pi} K_{s n-s}(\nu) d \nu=1$. Thus,

$$
K_{s n-s}(\nu)=\frac{1}{2}+\sum_{k=1}^{s n-s} \rho_{k, s n-s} \cos k \nu .
$$

The degree of approximation by convolution operators of the above form and by some of their modifications was recently investigated in a series of papers by the present authors (see [5]-[12], [18]-[19]). One quantitative result needed below is the following.

LEMMA 1.2. (Cao [5, Theorem 1], [6]). Let $n \in \mathbb{N}$ and $K_{m(n)}(v) \geq 0$. Then for $-1 \leq x \leq 1$ and $f \in C[-1,1]$,

$$
\left|f(x)-G_{m(n)}(f, x)\right| \leq 2 \omega_{1}\left[f ;\left(1-\rho_{1, m(n)}\right) \cdot|x|+\sqrt{2} \cdot \sqrt{1-\rho_{1, m(n)}} \cdot \sqrt{1-x^{2}}\right] .
$$

Here $\omega_{1}(f ; \cdot)$ is the first order modulus of continuity of $f$.

Shape preservation properties of an operator can be expressed using its behavior on the cones of $j$-convex functions. Let $j \in \mathbb{N}$ and $f \in C[a, b]$. The $j$-th forward difference of $f$ with increment $h$ is then given by

$\Delta_{h}^{j} f(t):=\sum_{k=0}^{j}(-1)^{j-k}\left(\begin{array}{l}j \\ k\end{array}\right) f(t+k h), \quad 0 \leq h \leq(b-a) / j$ and $t \in[a, b-j h]$.

A function $f$ is called $j$-convex if $f \in C[a, b]$ and all $j$-th forward differences $\Delta_{h}^{j} f(t), 0 \leq h \leq(b-a) / j$, are non-negative. Also, the function $f$ is said to be 0 -convex if it is non-negative on $[a, b]$.

As far as shape preservation by convolution operators of the above form is concerned, the following result is important. 
LEMMA 1.3. (Beatson [1, Theorem 1]). Let $f, g \in C[-1,1]$, and let the convolution operator $G$ be given by

$$
G(f, x)=(f * g)(x)=\frac{1}{\pi} \int_{-\pi}^{\pi} f(\cos \nu) g[\cos (\theta-\nu)] d \nu, \quad x=\cos \theta .
$$

Let $j$ be a non-negative integer. Then the cone of $j$-convex functions is invariant under the operator $G(f)=f * g$ if and only if $g$ is $j$-convex.

For the case $j=1$, this result had been obtained earlier by Roulier [28] and Senderovizh [29]. Based upon this lemma Beatson proved the following.

THEOREM B. (Beatson [1, Theorem 2]). Let $j$ be a positive integer. There exists an $M_{j}$ such that for each $f \in C[-1,1]$ and $n=0,1,2, \ldots$, there exists a $P_{n} \in \Pi_{n}$ with $P_{n} i$-convex for any $i \in\{0,1, \ldots\}$ for which $f$ is $i$-convex and

$$
\left|f(x)-P_{n}(x)\right| \leq M_{j} \omega_{1}\left[f, \Delta_{n}(x)\right], \quad|x| \leq 1 .
$$

Here, $\Delta_{0}(x):=1$ and, for $n \geq 1, \Delta_{n}(x):=\max \left(\sqrt{1-x^{2}} / n, 1 / n^{2}\right)$.

For the case $j=1$, a pointwise theorem had been obtained earlier by Lorentz and Zeller [25, Theorem 2], and for $j=2$ a uniform estimate of the above type was first given by Shvedov [30, Theorem 4]. Theorem B shows that, in a Timan-type theorem, cones of $i$-convex functions can be assumed to be invariant up to some predescribed order $j$.

However, for certain cases it is known that $\omega_{1}\left[f, \Delta_{n}(x)\right]$ can be replaced by the quantity $\omega_{2}\left(f, \sqrt{1-x^{2}} / n\right)$.

THEOREM C. (DeVore and $Y u$ [16]). Let $f \in C[-1,1]$ be an increasing function. Then there exists $P_{n}(f, \cdot) \in \Pi_{n}$ such that $P_{n}(f, \cdot)$ is increasing and

$$
\left|f(x)-P_{n}(f, x)\right| \leq c \cdot \omega_{2}\left(f, \sqrt{1-x^{2}} / n\right), \quad|x| \leq 1 .
$$

Here the constant $c$ is independent of $f, x$ and $n$.

THEOREM D. (Yu [32], Leviatan [22]). If $f \in C[-1,1]$ is a convex function then there is a convex polynomial $P_{n} \in \Pi_{n}$, such that

$$
\left|f(x)-P_{n}(f, x)\right| \leq c \cdot \omega_{2}\left(f, \sqrt{1-x^{2}} / n\right), \quad|x| \leq 1,
$$

with $c$ independent of $f, x$ and $n$. 
Uniform results corresponding to the pointwise ones in Theorems C and D had been obtained earlier by Shvedov [31, Theorem 1].

In the present note we shall investigate this matter further using the Boolean sum approach which was also employed in our other papers mentioned. To be more specific, below we will first construct and study certain convolution operators $H_{n, s, j}$ based upon modified Matsuoka kernels to generalize Beatson's Theorem B (see Theorem 4.3 below). Furthermore, we shall use Boolean sum modifications of $H_{n, s, j}$ in order to investigate the invariance of cones of $i$-convex functions in Telyakovskii and Gopengauz-type estimates (Theorems 4.4 and 4.5). As special cases we obtain Theorems C and D. In the final Section 5 we briefly discuss a further type of Boolean sum modification and show that for this one similar results can be derived.

We make the following additional remarks concerning notation in this paper. For $f \in C[a, b]$, let $\|f\|:=\max \{|f(t)|: a \leq t \leq b\}$. By $C, \widetilde{C}$ (upper or lower case) we will denote positive absolute constants independent of $n, f$ and $x \in[a, b]$. The constants $C$ and $\widetilde{C}$ may be different at different occurrences, even on the same line. Occasionally they will carry subscripts in order to explicitly indicate the quantities they depend on.

\section{Further notes on the Boolean sum method}

In this section we prove several assertions concerning Boolean sums of certain positive linear operators. Although the results of this section are mainly of an auxiliary nature for the main theorems of later sections, they appear to be of independent interest.

Let $L f$ be the linear function interpolating $f$ at $a$ and $b$, that is,

$$
L(f, x)=\frac{f(b)(x-a)+f(a)(b-x)}{b-a}, \quad a \leq x \leq b .
$$

Let $A$ be a linear operator mapping $C[a, b]$ into $C[a, b]$, and let $A^{+}$denote the Boolean sum of $L$ and $A$ given by

$$
\begin{aligned}
A^{+}(f, x) & :=(L \oplus A)(f, x)=L(f ; x)+A(f ; x)-(L \circ A)(f ; x) \\
& =A(f, x)+\{(x-a)[f(b)-A(f, b)]+(b-x)[f(a)-A(f, a)]\} /(b-a) .
\end{aligned}
$$

LEMMA 2.1. Let $A$ be a positive linear operator mapping $C[a, b]$ into itself, and such that $A(1 ; x)=1$. Let $f \in C[a, b]$ be increasing, and

$$
v(x):=\{(x-a)[f(b)-A(f, b)]+(b-x)[f(a)-A(f, a)]\} /(b-a) .
$$

Then $v$ also increases on $[a, b]$ 
Proof. Since $f$ is increasing on $[a, b]$, we have $f(a) \leq f(x) \leq f(b), a \leq x \leq b$. The operator $A$ is positive, so $A(f-f(a) ; x) \geq 0$, which implies $A(f ; x)-f(a)$. $A(1 ; x) \geq 0$. In particular, $A(f ; a)-f(a) \geq 0$. Similarly, $f(b)-A(f ; b) \geq 0$.

Thus

$$
\nu^{\prime}(x):=\{[f(b)-A(f, b)]+[A(f ; a)-f(a)]\} /(b-a) \geq 0, \quad x \in[a, b],
$$

from where it follows that $v$ increases on $[a, b]$.

LEMMA 2.2. Let $A$ be a positive linear operator as in Lemma 2.1. For $j \in \mathbb{N}$, let the cone of $j$-convex functions be invariant under the operator $A$. Then the cone of $j$-convex functions is also invariant under the operator $A^{+}$.

PROOF. If $j=1$, and $f \in C[a, b]$ is increasing on $[a, b]$, by Lemma 2.1 it follows that the function $v(x)$ is increasing, that is, $\Delta_{h}^{1} v(x) \geq 0,0 \leq h \leq b-a, x \in[a, b-h]$. Under the conditions of Lemma 2.2, we have $\Delta_{h}^{1} A(f, x) \geq 0$. Since $A^{+}(f, x)=$ $A(f, x)+v(x)$, we have that

$$
\Delta_{h}^{1} A^{+}(f, x)=\Delta_{h}^{1} A(f, x)+\Delta_{h}^{1} \nu(x) \geq 0 .
$$

If $j \geq 2$, then $\Delta_{h}^{j}(\alpha x+\beta)=0$, and thus

$$
\Delta_{h}^{j} A^{+}(f, x)=A(f, x)+\Delta_{h}^{j} \nu(x)=A(f, x) .
$$

Hence, if $\Delta_{h}^{j} f(x) \geq 0$, from the assumption of Lemma 2.2 we have that

$$
\Delta_{h}^{j} A^{+}(f, x)=\Delta_{h}^{j} A(f, x) \geq 0 .
$$

In view of the result of Lemma 2.2 it is a natural question if the Boolean sum $A^{+}$of positive operators $L$ and $A$ is also positive. This is not true as can be seen by choosing

$$
\begin{gathered}
{[a, b]=[0,1], \quad L(f ; x)=f(1) \cdot x+f(0) \cdot(1-x),} \\
A(f ; x)=(x+1) \cdot f(x), \quad x \in[0,1] .
\end{gathered}
$$

Then both $L$ and $A$ are positive, and $A^{+}(f ; x)=(x+1) f(x)-x \cdot f(1) . A^{+}$is not positive. In fact, choose $f(x)=x^{2}, x \in[0,1]$. Then

$$
A^{+}\left(f ; \frac{1}{2}\right)=\frac{3}{2} \cdot f\left(\frac{1}{2}\right)-\frac{1}{2} f(1)=-\frac{1}{8}<0 .
$$

For operators $G_{m(n)}$ as introduced above the statement of the example below holds.

EXAMPLE 2.3. Let $K_{2}$ with $\rho_{2,2}>0,-1 \leq t \leq 1$ be a positive kernel. Then there is a function $g \in C[-1,1]$ such that $g(t) \geq 0,-1 \geq t \geq 1$, and $G_{2}^{+}(g, 0)<0$, that is, $G_{0}^{+}$is a non-positive linear operator.

We do not give a proof of Example 2.3 here; the reader is referred instead to the technical report [13], where full details are given. 


\section{Some auxiliary results}

In this section we collect several assertions of auxiliary nature (Lemmas 3.1 through 3.3). We also recall two results which were proved previously by the present authors (Lemmas 3.4 and 3.5).

Let $g \in C[a, b]$. We write $F_{0}(u):=g(u), u \in[a, b]$. For $r \in \mathbb{N}$ let $F_{r}(u)$ be the integral of $F_{r-1}$ on $[a, u]$. It can be shown that ([33, chapter XII, § 8])

$$
F_{r}(u)=\frac{1}{(r-1) !} \int_{a}^{u}(u-v)^{r-1} g(v) d v .
$$

Indeed, by differentiation with regard to the parameter $\mathrm{u}$ we see that, for $1 \leq i \leq r-1$,

$$
\frac{d^{i}}{d u^{i}} F_{r}(u)=F_{r-i}(u)=\frac{1}{(r-i-1) !} \int_{a}^{u}(u-v)^{r-i-1} g(v) d v
$$

and

$$
\frac{d^{r}}{d u^{r}} F_{r}(u)=g(u)
$$

In the sequel, let $M_{m, r}$ and $\bar{M}_{m, r}$ be positive constants depending on $m$ and $r$ only.

LEMMA 3.1. Let $m \geq 0, r \geq 1, g \in C[-1,1], g(z) \geq 0,-1 \leq z \leq 1$. Then

$$
\begin{aligned}
I_{m, r} & :=\int_{0}^{\pi} \nu^{m}\left(\int_{-1}^{\cos \nu}(\cos \nu-\xi)^{r-1} g(\xi) d \xi\right) d \nu \\
& \leq M_{m, r} \int_{0}^{\pi} v^{2 r+m} g(\cos v) d v .
\end{aligned}
$$

PROOF. We proceed by induction for $r=1,2, \ldots$ :

If $r=1$, integration by parts gives

$$
\begin{aligned}
I_{m, 1} & =\int_{0}^{\pi} \nu^{m}\left(\int_{-1}^{\cos \nu} g(\xi) d \xi\right) d \nu \\
& =\left.\left[\frac{v^{m+1}}{m+1}\left(\int_{-1}^{\cos \nu} g(\xi) d \xi\right)\right]\right|_{\nu=0} ^{\nu=\pi}+\frac{1}{m+1} \int_{0}^{\pi} \nu^{m+1} \sin \nu \cdot g(\cos \nu) d \nu \\
& \leq \frac{1}{m+1} \int_{0}^{\pi} \nu^{m+2} g(\cos \nu) d \nu,
\end{aligned}
$$

since $\sin v \leq \nu, 0 \leq \nu \leq \pi$.

Assume (3.4) holds for $r=r_{0}$ and any $m=0,1,2, \ldots$. Another integration by parts yields

$$
I_{m, r_{0}+1}=\int_{0}^{\pi} \nu^{m}\left(\int_{-1}^{\cos \nu}(\cos \nu-\xi)^{r_{0}} g(\xi) d \xi\right) d \nu
$$




$$
\begin{aligned}
& =\left.\left[\frac{\nu^{m+1}}{m+1}\left(\int_{-1}^{\cos \nu}(\cos \nu-\xi)^{r_{0}} g(\xi) d \xi\right)\right]\right|_{\nu=0} ^{\nu=\pi} \\
& \quad-\frac{1}{m+1} \int_{0}^{\pi} v^{m+1}\left(\int_{-1}^{\cos \nu}(\cos \nu-\xi)^{r_{0}} g(\xi) d \xi\right)^{\prime} d \nu \\
& =-\frac{1}{m+1} \int_{0}^{\pi} v^{m+1}\left(\int_{-1}^{\cos \nu}(\cos v-\xi)^{r_{0}} g(\xi) d \xi\right)^{\prime} d \nu,
\end{aligned}
$$

where the prime denotes differentiation with respect to $\nu$.

From (3.2), we know that

$$
\frac{1}{r_{0} !} \frac{d}{d u}\left(\int_{-1}^{u}(u-\xi)^{r_{0}} g(\xi) d \xi\right)=\frac{1}{\left(r_{0}-1\right) !} \int_{-1}^{u}(u-\xi)^{r_{0}-1} g(\xi) d \xi
$$

whence, for the derivative figuring in (3.6), we have

$$
\frac{d}{d \nu}\left(\int_{-1}^{\cos \nu}(\cos v-\xi)^{r_{0}} g(\xi) d \xi\right)=-r_{0} \int_{-1}^{\cos v}(\cos \nu-\xi)^{r_{0}-1} \sin \nu \cdot g(\xi) d \xi
$$

Thus

$$
\begin{aligned}
I_{m, r_{0}+1} & =\frac{r_{0}}{m+1} \int_{0}^{\pi} v^{m+1}\left(\int_{-1}^{\cos \nu}(\cos v-\xi)^{r_{0}-1} \sin v g(\xi) d \xi\right) d v \\
& \leq \frac{r_{0}}{m+1} \int_{0}^{\pi} v^{m+2}\left(\int_{-1}^{\cos \nu}(\cos v-\xi)^{r_{0}-1} g(\xi) d \xi\right) d \nu \\
& =\frac{r_{0}}{m+1} I_{m+2, r_{0}} \\
& \leq \frac{r_{0}}{m+1} M_{m+2, r_{0}} \int_{0}^{\pi} v^{2 r_{0}+m+2} g(\cos v) d v \\
& =\frac{r_{0}}{m+1} M_{m+2, r_{0}} \int_{0}^{\pi} v^{2\left(r_{0}+1\right)+m} g(\cos v) d \nu .
\end{aligned}
$$

Hence (3.4) holds for $r=r_{0}+1$ and the lemma is proved.

The next lemma gives an estimate from below.

LEMMA 3.2. Let $m \geq 0, r \geq 1, g \in C[-1,1], g(z) \geq 0,-1 \leq z \leq 1$. Then

$$
\begin{aligned}
J_{m, r} & :=\int_{0}^{\pi / 2} v^{m}\left(\int_{-1}^{\cos \nu}(\cos v-\xi)^{r-1} g(\xi) d \xi\right) d \nu \\
& \geq \bar{M}_{m, r} \int_{0}^{\pi / 2} v^{2 r+m} g(\cos v) d \nu
\end{aligned}
$$


PROOF. We apply again induction on $r$. If $r=1$, integration by parts shows that

$$
\begin{aligned}
J_{m, 1} & =\int_{0}^{\pi / 2} \nu^{m}\left(\int_{-1}^{\cos \nu} g(\xi) d \xi\right) d \nu \\
& =\left.\frac{\nu^{m+1}}{m+1}\left(\int_{-1}^{\cos \nu} g(\xi) d \xi\right)\right|_{\nu=0} ^{\nu=\pi / 2}+\frac{1}{m+1} \int_{0}^{\pi / 2} \nu^{m+1} \sin v g(\cos v) d v \\
& \geq \frac{(\pi / 2)^{m+1}}{m+1}\left(\int_{1}^{0} g(\xi) d \xi\right)+\frac{2}{\pi(m+1)} \int_{0}^{\pi / 2} \nu^{m+2} g(\cos v) d v \\
& \geq \frac{2}{\pi(m+1)} \int_{0}^{\pi / 2} v^{m+2} g(\cos v) d \nu,
\end{aligned}
$$

since $(2 / \pi) \nu \leq \sin \nu, 0 \leq \nu \leq \pi / 2$. Let (3.8) hold for $r=r_{0}$ and any $m=0,1,2, \ldots$ Consider

$$
J_{m, r_{0}+1}=\int_{0}^{\pi / 2} \nu^{m}\left(\int_{-1}^{\cos v}(\cos v-\xi)^{r_{0}} g(\xi) d \xi\right) d \nu .
$$

Integrating by parts and using (3.7), we have

$$
\begin{aligned}
J_{m, r_{0}+1}= & \left.\frac{\nu^{m+1}}{m+1} \nu^{m}\left(\int_{-1}^{\cos \nu}(\cos \nu-\xi)^{r_{0}} g(\xi) d \xi\right)\right|_{\nu=0} ^{\nu=\pi / 2} \\
& \quad-\frac{1}{m+1} \int_{0}^{\pi / 2} \nu^{m+1}\left(\int_{-1}^{\cos \nu}(\cos \nu-\xi)^{r_{0}} g(\xi) d \xi\right)^{\prime} d \nu \\
= & \frac{(\pi / 2)^{m+1}}{m+1}\left(\int_{-1}^{0}(0-\xi)^{r_{0}} g(\xi) d \xi\right) \\
& +\frac{r_{0}}{m+1} \int_{0}^{\pi / 2} \nu^{m+1} \sin \nu\left(\int_{-1}^{\cos \nu}(\cos \nu-\xi)^{r_{0}-1} g(\xi) d \xi\right) d \nu \\
\geq & \frac{2 r_{0}}{\pi(m+1)} \int_{0}^{\pi / 2} v^{m+2}\left(\int_{-1}^{\cos \nu}(\cos \nu-\xi)^{r_{0}-1} g(\xi) d \xi\right) d \nu \\
\geq & \frac{2 r_{0}}{\pi(m+1)} \bar{M}_{m+2, r_{0}} \int_{0}^{\pi / 2} v^{2 r_{0}+m+2} g(\cos \nu) d \nu \\
= & \frac{2 r_{0}}{\pi(m+1)} \bar{M}_{m+2, r_{0}} \int_{0}^{\pi / 2} \nu^{2\left(r_{0}+1\right)+m} g(\cos \nu) d \nu .
\end{aligned}
$$

This yields the claim of Lemma 3.2.

LEMMA 3.3. Let $p$ and $q \in \mathbb{N}, p \geq q$. Then

$$
\int_{0}^{\pi} \frac{(\sin (n v / 2))^{2 p}}{v^{2 q}} d \nu \approx n^{2 q-1}, \quad \int_{0}^{\pi / 2} \frac{(\sin (n v / 2))^{2 p}}{v^{2 q}} d \nu \approx n^{2 q-1}
$$

Here, $a_{n} \approx b_{n}$ if and only if $a_{n}=O\left(b_{n}\right)$ and $b_{n}=O\left(a_{n}\right)$. 
PROOF. See [15, p. 80].

LEMMA 3.4. Let $n \geq 1, m(n) \in \mathbb{N} \cup\{0\}$ with $c n \leq m(n) \leq \tilde{c} n$ for $n \geq 2$ and for some constants $c, \tilde{c}$. Let $A_{n}: C[-1,1] \rightarrow \Pi_{m(n)}$ be a sequence of linear algebraic polynomial operators. Suppose that for $A_{n}$ we have the Timan-type estimate

$$
\left|A_{n}(f, x)-f(x)\right| \leq c \omega_{1}\left(f, \sqrt{1-x^{2}} / n, 1 / n^{2}\right), \quad|x| \leq 1 .
$$

Then for $A_{n}^{+}$, the Telyakovskil-type estimate

$$
\left|A_{n}^{+}(f, x)-f(x)\right| \leq c \omega_{1}\left(f, \sqrt{1-x^{2}} / n\right), \quad|x| \leq 1,
$$

holds true.

PROOF. See Cao and Gonska [12, Theorem 3].

LEMMA 3.5. Let $n \geq 2$ and $c_{n} \leq m(n) \leq \tilde{c}$ for some constants $c, \tilde{c}$. Furthermore, let $K_{m(n)}(v) \geq 0$, and

(i) $1-\rho_{1, m(n)}=O\left(n^{-2}\right)$,

(ii) $3 / 2-2 \rho_{1, m(n)}+1 / 2 \rho_{2, m(n)}=O\left(n^{-4}\right)$.

Then, for all $f \in C[-1,1]$,

$$
\left|G_{m(n)}^{+}(f, x)-f(x)\right| \leq c \omega_{2}\left(f, \sqrt{1-x^{2}} / n\right), \quad|x| \leq 1 .
$$

Proof. See Cao and Gonska [8, Theorem 5.4].

\section{Approximation by Boolean sums $G_{m(n)}^{+}$.}

We start this section with the construction of certain kernels $\bar{F}_{n, s, j}(v)$ upon which the definition of shape preserving operators $H_{n, s, j}$ will be based.

Let $\xi=\cos (v)$. Starting from (1.4), we define

$$
K_{s n-n}(\arccos \xi)=C_{n, s}\left(\frac{\sin (n \arccos \xi / 2)}{\sin (\arccos \xi / 2)}\right)^{2 s}=\frac{1}{2}+\sum_{k=1}^{s n-s} \rho_{k, s n-s} \cos (k \arccos \xi) .
$$

Clearly $K_{s n-s}(\arccos \xi) \in \Pi_{s n-s}$.

For $j \in \mathbb{N}$, we define

$$
F_{n, s, j}(z):=\frac{1}{(j-1) !} \int_{-1}^{z}(z-\xi)^{j-1} K_{s n-s}(\arccos \xi) d \xi
$$


to be a $j$-th antiderivative of $K_{s n-s}(\arccos \xi)$; thus $F_{n, s, j} \in \Pi_{s n-s+j}$, and

$$
F_{n, s, j}(z)=\tau_{0, n, s, j}+\tau_{1, n, s, j} \cdot z+\cdots+\tau_{s n-s+j, n, s, j} \cdot z^{s n-s+j},
$$

or

$$
F_{n, s, j}(\cos \nu)=\tau_{0, n, s, j}+\tau_{1, n, s, j} \cos \nu+\cdots+\tau_{s n-s+j, n, s, j}(\cos \nu)^{s n-s+j}
$$

Normalizing yields the kernel

$$
\bar{F}_{n, s, j}(\nu):=\frac{\pi F_{n, s, j}(\cos v)}{\int_{-\pi}^{\pi} F_{n, s, j}(\cos t) d t},
$$

for which

$$
\frac{1}{\pi} \int_{-\pi}^{\pi} \bar{F}_{n, s, j}(\nu) d v=1
$$

Furthermore, from (4.2) we have

$\bar{F}_{n, s, j}(v)=\lambda_{0, n, s, j}+\lambda_{1, n, s, j} \cos v+\lambda_{2, n, s, j} \cos 2 v+\cdots+\lambda_{s n-s+j, n, s, j} \cos (s n-s+j) v$.

Since

$$
\frac{1}{\pi} \int_{-\pi}^{\pi} \bar{F}_{n, s, j}(v) d v=1=2 \cdot \lambda_{0, n, s, j},
$$

we have $\lambda_{o, n, s, j}=\frac{1}{2}$. We denote the convolution-type operators (1.3) with kernel $\bar{F}_{n, s, j}(\nu)$ by $H_{n, s, j}$. Then

$$
H_{n, s, j}(1, x)=\pi^{-1} \int_{-\pi}^{\pi} \bar{F}_{n, s, j}(v) d v=1
$$

and, from (4.5),

$$
\lambda_{k, n, s, j}=\frac{1}{\pi} \int_{-\pi}^{\pi} \cos k \nu \bar{F}_{n, s, j}(\nu) d \nu, \quad 1 \leq k \leq s n-s+j .
$$

The next lemma shows that the coefficients of $\cos v$ in (4.5) are sufficiently close to 1 .

LEMMA 4.1. Let $j \in \mathbb{N}$ and $s \geq j+2$. Then

$$
1-\lambda_{1, n, s, j}=O\left[n^{-2}\right]
$$


PROOF. From (4.6), (4.7), and (4.3), we have

$$
\begin{aligned}
1-\lambda_{1, n, s, j} & =\frac{1}{\pi} \int_{-\pi}^{\pi}(1-\cos \nu) \bar{F}_{n, s, j}(\nu) d \nu \\
& =\frac{1}{\pi} \int_{-\pi}^{\pi}(1-\cos \nu)\left[\frac{\pi \cdot F_{n, s, j}(\cos \nu)}{\int_{-\pi}^{\pi} F_{n, s, j}(\cos t) d t}\right] d \nu \\
& =\frac{1}{\int_{-\pi}^{\pi} F_{n, s, j}(\cos \nu) d \nu} \times \int_{-\pi}^{\pi} 2 \sin ^{2} \frac{\nu}{2} F_{n, s, j}(\cos \nu) d \nu \\
& \leq \frac{1}{2} \frac{\int_{-\pi}^{\pi} v^{2} F_{n, s, j}(\cos \nu) d \nu}{\int_{-\pi}^{\pi} F_{n, s, j}(\cos v) d \nu} .
\end{aligned}
$$

Using the inequality

$$
\frac{2}{\pi} \nu \leq \sin \nu, \quad 0 \leq v \leq \frac{\pi}{2}
$$

we have, on the other hand,

$$
1-\lambda_{1, n, s, j} \geq \frac{2}{\pi^{2}} \frac{\int_{-\pi}^{\pi} v^{2} F_{n, s, j}(\cos v) d \nu}{\int_{-\pi}^{\pi} F_{n, s, j}(\cos v) d \nu} .
$$

Let $N_{r}, r=1,2, \ldots$, be positive constants independent of $n$. Write

$$
\begin{aligned}
\int_{-\pi}^{\pi} F_{n, s, j}(\cos \nu) d \nu & =2 \int_{0}^{\pi} F_{n, s, j}(\cos \nu) d \nu \\
& =\frac{2}{(j-1) !} \int_{0}^{\pi}\left(\int_{-1}^{\cos \nu}(\cos \nu-\xi)^{j-1} K_{s n-s}(\arccos \xi) d \xi\right) d \nu \\
& =: R_{n, s, j} .
\end{aligned}
$$

Using Lemma 3.1 (for the case $m=0, r=j$ ), we get

$$
\begin{aligned}
R_{n, s, j} & \leq \frac{2 M_{0, j}}{(j-1) !} \int_{0}^{\pi} v^{2 j} K_{s n-s}[\arccos (\cos v)] d v \\
& =N_{1} \int_{0}^{\pi} v^{2 j} K_{s n-s}(\nu) d \nu .
\end{aligned}
$$

Using Lemma 3.2 (again for the case $m=0, r=j$ ) we have

$$
\begin{aligned}
R_{n, s, j} & \geq \frac{2 \bar{M}_{0, j}}{(j-1) !} \int_{0}^{\pi / 2} v^{2 j} K_{s n-s}[\arccos (\cos \nu)] d \nu \\
& =N_{2} \int_{0}^{\pi / 2} v^{2 j} K_{s n-s}(\nu) d \nu .
\end{aligned}
$$


Define

$$
\begin{aligned}
\bar{R}_{n, s, j} & :=\int_{-\pi}^{\pi} v^{2} F_{n, s, j}(\cos v) d \nu \\
& =2 \int_{0}^{\pi} v^{2} F_{n, s, j}(\cos v) d v \\
& =\frac{2}{(j-1) !} \int_{0}^{\pi} \nu^{2}\left(\int_{-1}^{\cos v}(\cos v-\xi)^{j-1} K_{s n-s}(\arccos \xi) d \xi\right) d \nu
\end{aligned}
$$

Again, using Lemma 3.1, we derive

$$
\bar{R}_{n, s, j} \leq N_{3} \int_{0}^{\pi} v^{2 j+2} K_{s n-s}(\nu) d \nu
$$

and using Lemma 3.2, we have

$$
\bar{R}_{n, s, j} \geq N_{4} \int_{0}^{\pi / 2} v^{2 j+2} K_{s n-s}(v) d v
$$

Combining (4.8), (4.10), (4.11), (4.12), (4.13) and (4.14), we obtain

$$
1-\lambda_{1, n, s, j} \leq N_{5} \frac{\int_{0}^{\pi} v^{2 j+2} K_{s n-s}(v) d v}{\int_{0}^{\pi / 2} v^{2 j} K_{s n-s}(v) d v}
$$

and

$$
1-\lambda_{1, n, s, j} \geq N_{6} \frac{\int_{0}^{\pi / 2} v^{2 j+2} K_{s n-s}(\nu) d v}{\int_{0}^{\pi} v^{2 j} K_{s n-s}(\nu) d v}
$$

By Lemma 3.3, we have

$$
\begin{aligned}
\int_{0}^{\pi} v^{2 j+2} K_{s n-s}(\nu) d v & =C_{n, s} \int_{0}^{\pi} v^{2 j+2}\left(\frac{\sin \frac{n v}{2}}{\sin \frac{v}{2}}\right)^{2 s} d \nu \\
& \approx C_{n, s} \int_{0}^{\pi} v^{2 j+2} \frac{\left(\sin \frac{n v}{2}\right)^{2 s}}{v^{2 s}} d v \\
& =C_{n, s} \int_{0}^{\pi} \frac{\left(\sin \frac{n \nu}{2}\right)^{2 s}}{v^{2(s-j-1)}} d v \\
& \approx C_{n, s} \cdot n^{2(s-j-1)-1}, \quad s \geq j+2
\end{aligned}
$$


Similarly,

$$
\begin{array}{cc}
\int_{0}^{\pi / 2} v^{2 j+2} K_{s n-s}(v) d v \approx C_{n, s} \cdot n^{2(s-j-1)-1}, & s \geq j+2, \\
\int_{0}^{\pi} v^{2 j} K_{s n-s}(v) d v \approx C_{n, s} \cdot n^{2(s-j)-1}, & s \geq j+1, \\
\int_{0}^{\pi / 2} v^{2 j} K_{s n-s}(v) d v \approx C_{n, s} \cdot n^{2(s-j)-1}, & s \geq j+1 .
\end{array}
$$

Combining (4.15) through (4.20), we obtain that if $s \geq j+2$, then

$$
1-\lambda_{1, n, s, j} \approx \frac{n^{2(s-j-1)-1}}{n^{2(s-j)-1}}=O\left(n^{-2}\right)
$$

LEMMA 4.2. Let $j \in \mathbb{N}$ and $s \geq j+3$. Then

$$
\frac{3}{2}-2 \lambda_{1, n, s, j}+\frac{1}{2} \lambda_{2, n, s, j}=O\left(n^{-4}\right) .
$$

Proof. From (4.6), (4.7), and (4.3), we have

$$
\begin{aligned}
\frac{3}{2}-2 \lambda_{1, n, s, j}+\frac{1}{2} \lambda_{2, n, s, j} & =\pi^{-1} \int_{-\pi}^{\pi}\left(1-2 \cos \nu+\frac{1}{2}(1+\cos 2 v)\right) \bar{F}_{n, s, j}(\nu) d \nu \\
& =\pi^{-1} \int_{-\pi}^{\pi}\left(1-2 \cos \nu+\cos ^{2} \nu\right) \bar{F}_{n, s, j}(\nu) d \nu \\
& =\pi^{-1} \int_{-\pi}^{\pi}(1-\cos \nu)^{2} \bar{F}_{n, s, j}(\nu) d \nu \\
& =\int_{-\pi}^{\pi}(1-\cos v)^{2}\left[\frac{F_{n, s, j}(\cos v)}{\int_{-\pi}^{\pi} F_{n, s, j}(\cos t) d t}\right] d \nu \\
& =\frac{\int_{-\pi}^{\pi} 4 \sin ^{4} \frac{\nu}{2} F_{n, s, j}(\cos v) d \nu}{\int_{-\pi}^{\pi} F_{n, s, j}(\cos v) d \nu}
\end{aligned}
$$

From Lemmas 3.1 and 3.2, using a method similar to the proofs of (4.15) and (4.16), we obtain

$$
\frac{3}{2}-2 \lambda_{1, n, s, j}+\frac{1}{2} \lambda_{2, n, s, j} \leq N_{7} \frac{\int_{0}^{\pi} v^{2 j+4} K_{s n-s}(\nu) d \nu}{\int_{0}^{\pi / 2} v^{2 j} K_{s n-s}(\nu) d \nu}
$$


as well as

$$
\frac{3}{2}-2 \lambda_{1, n, s, j}+\frac{1}{2} \lambda_{2, n, s, j} \geq N_{8} \frac{\int_{0}^{\pi / 2} v^{2 j+4} K_{s n-s}(v) d v}{\int_{0}^{\pi} v^{2 j} K_{s n-s}(v) d v}
$$

By Lemma 3.3 we have

$$
\begin{aligned}
\int_{0}^{\pi} v^{2 j+4} K_{s n-s}(\nu) d v & =C_{n, s} \int_{0}^{\pi} v^{2 j+4}\left(\frac{\sin (n v / 2)}{\sin (v / 2)}\right)^{2 s} d v \\
& \approx C_{n, s} \int_{0}^{\pi} v^{2 j+4} \frac{(\sin (n v / 2))^{2 s}}{v^{2 s}} d v \\
& =C_{n, s} \int_{0}^{\pi} \frac{(\sin (n v / 2))^{2 s}}{v^{2(s-j-2)}} d v \\
& \approx C_{n, s} \cdot n^{2(s-j-2)-1}, \quad s \geq j+3 .
\end{aligned}
$$

Similarly,

$$
\int_{0}^{\pi / 2} v^{2 j+4} K_{s n-s}(v) d v \approx C_{n, s} \cdot n^{2(s-j-2)-1}, \quad s \geq j+3 .
$$

From (4.19) and (4.20), we have

$$
\begin{array}{ll}
\int_{0}^{\pi} v^{2 j} K_{s n-s}(\nu) d \nu \approx C_{n, s} \cdot n^{2(s-j)-1}, & s \geq j+1 \\
\int_{0}^{\pi / 2} \nu^{2 j} K_{s n-s}(\nu) d v \approx C_{n, s} \cdot n^{2(s-j)-1}, & s \geq j+1 .
\end{array}
$$

Combining (4.23) through (4.28), we obtain the result that if $s \geq j+3$, then

$$
\frac{3}{2}-2 \lambda_{1, n, s, j}+\frac{1}{2} \lambda_{2, n, s, j} \approx \frac{n^{2(s-j-2)-1}}{n^{2(s-j)-1}}=O\left(n^{-4}\right) .
$$

The following Theorems 4.3 through 4.5 constitute the main results of this note. Our first theorem deals with the quantitative and shape preserving properties of the operators $H_{n, s, j}$ based upon the kernels $\bar{F}_{n, s, j}$ (recall (4.5) and its neighbourhood). Note that shape, as expressed by $i$-convexity, is fully retained by the $H_{n, s, j}$, including positivity. This will be different in Theorems 4.4 and 4.5 .

THEOREM 4.3. Let $j \in \mathbb{N}$ and $s \geq j+2$. There exists a positive constant $c_{j, s}$ such that, for each $f \in C[-1,1]$ and $n \geq 1$, the polynomial $H_{n, s, j}(f, \cdot) \in \Pi_{s n-s+j}$ satisfies the inequality

$$
\left|f(x)-H_{n, s, j}(f, x)\right| \leq c_{j, s} \omega_{1}\left(f, \frac{\sqrt{1-x^{2}}}{n}+\frac{1}{n^{2}}\right), \quad|x| \leq 1 .
$$


Furthermore, if the function $f$ is $i$-convex, then $H_{n, s, j}(f, \cdot)$ is also $i$-convex, where $i \in\{0,1, \ldots, j\}$.

PROOF. For the convolution-type operators $H_{n, s, j}$ with kernels $\bar{F}_{n, s, j} \geq 0$, using Lemma 4.1 we have

$$
1-\lambda_{1, n, s, j}=O\left(n^{-2}\right), \quad s \geq j+2 .
$$

Lemma 1.2 implies the Timan-type estimate

$$
\left|f(x)-H_{n, s, j}(f, x)\right| \leq c_{j, s} \omega_{1}\left(f, \frac{\sqrt{1-x^{2}}}{n}+\frac{1}{n^{2}}\right), \quad|x| \leq 1 .
$$

Since $H_{n, s, j}$ is a positive linear operator, we obtain for $i=0$ that, if $f \geq 0$, then $H_{n, s, j}(f, \cdot) \geq 0$. Furthermore, from (1.2) and (4.3), (4.1) we have

$$
\begin{aligned}
H_{n, s, j}(f, x) & =\frac{1}{\pi} \int_{-\pi}^{\pi} f(\cos \nu) \bar{F}_{n, s, j}(\theta-\nu) d \nu \\
& =\frac{1}{\int_{-\pi}^{\pi} F_{n, s, j}(\cos \nu) d \nu} \int_{-\pi}^{\pi} f(\cos v) F_{n, s, j}[\cos (\theta-\nu)] d \nu,
\end{aligned}
$$

where

$$
F_{n, s, j}(z):=\frac{1}{(j-1) !} \int_{-1}^{z}(z-\xi)^{j-1} K_{s n-s}(\arccos \xi) d \xi
$$

Using (3.2) and (3.3), we have, for $1 \leq i \leq j-1$, the representations

$$
\frac{d^{i}}{d z^{i}} F_{n, s, j}(z)=\frac{1}{(j-i-1) !} \int_{-1}^{z}(z-\xi)^{j-i-1} K_{s n-s}(\arccos \xi) d \xi \geq 0
$$

and

$$
\frac{d^{j}}{d z^{j}} F_{n, s, j}(z)=K_{s n-s}(\arccos z) \geq 0 .
$$

It is known (see [21]) that

$$
\Delta_{h}^{i} F_{n, s, j}(z)=F_{n, s, j}^{(i)}\left(\xi_{1}\right) h^{i} \geq 0,
$$

where $h \geq 0$ and $-1<\xi_{1}<1$, whence $F_{n, s, j}(z)$ is $i$-convex. Lemma 1.3 then implies that, if $f$ is $i$-convex, then so is $H_{n, s, j}(f, \cdot)$ for $i \in\{0,1, \ldots, j\}$.

From Theorem 4.3 we obtain Beatson's Theorem B and also the results by LorentzZeller [25] and Shvedov [30] mentioned earlier.

Our next theorem shows that extra interpolation conditions at the endpoints can be imposed. These will be achieved, however, at the expense of positivity. Note further that the following theorem only requires $s \geq j+2$. This is in contrary to the condition $s \geq j+3$ needed in Theorem 4.5 , and thus Theorem 4.4 is not a consequence of Theorem 4.5 . 
THEOREM 4.4. Let $j \in \mathbb{N}$ and $s \geq j+2$. Then there exists a positive constant $\tilde{c}_{j, s}$ such that for all $f \in C[-1,1]$ and $n \geq 1$, and $H_{n, s, j}^{+}(f, \cdot) \in \Pi_{s n-s+j}$,

$$
\left|f(x)-H_{n, s, j}^{+}(f, x)\right| \leq \tilde{c}_{j, s} \omega_{1}\left(f, \sqrt{1-x^{2}} / n\right) .
$$

Furthermore, if $f$ is $i$-convex, then $H_{n, s, j}^{+}(f, \cdot)$ is $i$-convex, where $i \in\{1, \ldots, j\}$.

ProOF. Since $s \geq j+2$, we have

$$
n \leq s n-s \leq s n-s+j \leq s n-s+s-2=s n-2 \leq s n, \quad n \geq 2, s \geq 3
$$

From Theorem 4.3, we know that

$$
\left|f(x)-H_{n, s, j}(f, x)\right| \leq c_{j, s} \omega_{1}\left(f, \frac{\sqrt{1-x^{2}}}{n}+\frac{1}{n^{2}}\right), \quad|x| \leq 1 .
$$

Using Lemma 3.4, we obtain

$$
\left|f(x)-H_{n, s, j}^{+}(f, x)\right| \leq \tilde{c}_{j, s} \omega_{1}\left(f, \frac{\sqrt{1-x^{2}}}{n}\right), \quad|x| \leq 1 .
$$

If $\mathrm{f}$ is $i$-convex, from Theorem 4.3 we find that $H_{n, s, j}(f, \cdot)$ is $i$-convex as well. Since $H_{n, s, j}(1, x)=1$ and $H_{n, s, j}$ is a positive linear operator, using Lemma 2.2 we obtain that $H_{n, s, j}^{+}(f, \cdot)$ is $i$-convex, too, where $i \in\{1, \ldots, j\}$.

From Theorem 4.4 again Beatson's Theorem B follows, but only for $i \in\{1, \ldots, j\}$. For $j=1$, we get again an estimate as that of Lorentz and Zeller, and for $j=2$ the uniform one of Shvedov.

Our final theorem in this section is formulated in terms of the second order modulus of smoothness.

THEOREM 4.5. Let $j \in \mathbb{N}$ and $s \geq j+3$. Then there exists a positive constant $c_{j . s}$ such that for each $f \in C[-1,1]$ and $n \geq 2$, and $H_{n, s, j}^{+}(f, \cdot) \in \Pi_{s n-s+j}$,

$$
\left|f(x)-H_{n, s, j}^{+}(f, x)\right| \leq c_{j, s} \omega_{2}\left(f, \sqrt{1-x^{2}} / n\right), \quad|x| \leq 1 .
$$

Also, if $f$ is $i$-convex, then $H_{n, s, j}^{+}(f, \cdot)$ is $i$-convex, too, where $i \in\{1, \ldots, j\}$.

Proof. First, if $f \in C[-1,1]$, then

$$
H_{n, s, j}(f, \cdot) \in \Pi_{s n-s+j} .
$$


Since $s \geq j+3$, we have

$$
n \leq s n-s+j \leq s n, \quad n \geq 2, s \geq 4 .
$$

For the operators $H_{n, s, j}$ based upon the kernels $\bar{F}_{n, s, j}(v) \geq 0$, using Lemmas 4.1 and 4.2 we have

$$
\begin{aligned}
1-\lambda_{1, n, s, j} & =O\left(n^{-2}\right), & & s \geq j+2, \\
\frac{3}{2}-2 \lambda_{1, n, s, j}+\lambda_{2, n, s, j} & =O\left(n^{-4}\right), & & s \geq j+3 .
\end{aligned}
$$

By Lemma 3.5, there exists a positive constant $c_{j, s}$ such that for $f \in C[-1,1]$ and $n \geq 2$,

$$
\left|f(x)-H_{n, s, j}^{+}(f, x)\right| \leq c_{j, s} \omega_{2}\left(f, \sqrt{1-x^{2}} / n\right), \quad|x| \leq 1 .
$$

By Theorem 4.4, the cone of $i$-convex functions is invariant under $H_{n, s, j}^{+}$, where $i \in\{1, \ldots, j\}$.

From Theorem 4.5 we again obtain Theorem B, where $i \in\{1, \ldots, j\}$. If $j=1$, we obtain Theorem $\mathrm{C}$, and for $j=2$, Theorem 4.5 implies Theorem $\mathrm{D}$ and the earlier uniform result by Shvedov [31, Theorem 1]. Furthermore, for the case $j=3$, we arrive at a refinement of another result by Shvedov [31, Theorem 2].

\section{Concluding remark: approximation by Boolean sums $G_{m(n)}^{*}$.}

In [7], we also investigated approximation properties of the Boolean sum

$$
\begin{aligned}
A^{*}(f, x) & :=(A \oplus L)(f ; x) \\
& =A(f ; x)+L(f ; x)-(A \circ L)(f ; x)=A(f-L f, x)+L(f, x) .
\end{aligned}
$$

In the applications given in [7] we had $A=G_{m(n)}$ (based upon suitable kernels $K_{m(n)}$ ), and $L$ was given as above.

If $G_{m(n)}=H_{n, s, j}$, with $H_{n, s, j}$ as given in Section 4, then the Boolean sums $H_{n, s, j}^{*}=$ $H_{n, s, j} \oplus L$ have a degree of approximation and preserve the shape of a function as indicated in the following theorem.

THEOREM 5.1. Let $j \in \mathbb{N}$ and $s \geq j+2$. There exists a positive constant $c_{j, s}$ such that for all $f \in C[-1,1], n \geq 1$, and $H_{n, s, j}^{*}(f, \cdot) \in \Pi_{s n-s+j}$,

$$
\left\|f-H_{n, s, j}^{*}(f, \cdot)\right\| \leq c_{j, s} \omega_{2}(f, 1 / n) .
$$

Furthermore, if $f$ is $i$-convex, then $H_{n, s, j}^{*}(f, \cdot)$ is $i$-convex, where $i \in\{1, \ldots, j\}$. 
Since we were only able to prove uniform inequalities involving $\omega_{2}(f, \cdot)$ for the operators $H_{n, s, j}^{*}$, we do not give a proof here. Details are contained in the technical report [13] available from the authors.

\section{References}

[1] R. K. Beatson, "Joint approximation of a function and its derivatives", in Approximation theory III (Proc. Int. Sympos. Austin 1980) (ed. E. W. Cheney), (Acad. Press, New York, 1980) 199-206.

[2] H. Berens and R. A. DeVore, "A characterization of Bernstein polynomials", in Approximation theory III (Proc. Int. Sympos. Austin 1980) (ed. E. W. Cheney), (Acad. Press, New York, 1980) 213-219.

[3] H. Berens and G. G. Lorentz, "Inverse theorems for Bernstein polynomials", Indiana Univ. Math. J. 21 (1972) 693-708.

[4] Jia-ding Cao, "On linear approximation methods (Chinese)", Acta Sci. Natur. Univ. Fudan 9 (1964) 43-52.

[5] Jia-ding Cao, "Generalization of Timan's theorem, Lehnhoff's theorem and Telyakovskiî's theorem”, Volume SM-DU-106 of Schriftenreihe des Fachbereichs Mathematik, (Universität Duisburg, 1986).

[6] Jia-ding Cao, "Generalizations of Timan theorem, Lehnhoff theorem and Telyakovskii theorem (Chinese)", Kexue Tongbao 15 (1986) 1132-1135, Science Bulletin (English Edition) 32 (1987), 1225-1229.

[7] Jia-ding Cao and H. H. Gonska, "Approximation by Boolean sums of positive linear operators", Rend. Mat. 6 (1986) 525-546.

[8] Jia-ding Cao and H. H. Gonska, "Approximation by Boolean sums of positive linear operators II: Gopengauz-type estimates", J. Approx. Theory 57 (1989) 77-89.

[9] Jia-ding Cao and H. H. Gonska, "Pointwise estimates for modified positive linear operators", Portugal. Math. 46 (1989) 401-430.

[10] Jia-ding Cao and H. H. Gonska, "Approximation by Boolean sums of positive linear operators III: Estimates for some numerical approximation schemes”, Numer. Funct. Anal. Optim. 10 (1989) 643-672.

[11] Jia-ding Cao and H. H. Gonska, "Computation of DeVore-Gopengauz-type approximants", in Approximation Theory VI (Proc. Int. Sympos. College Station 1989) (eds. C. K. Chui et al.), (Acad. Press, New York, 1989) 117-120.

[12] Jia-ding Cao and H. H. Gonska, "Approximation by Boolean sums of linear operators: Telyakovskil-type estimates”, Bull. Austral. Math. Soc. 42 (1990) 253-266.

[13] Jia-ding Cao and H. H. Gonska, "Approximation by Boolean sums of positive linear operators IV: shape preservation by DeVore-Gopengauz-type approximants", Volume SIAI-EBS-2-92 of Schriftenreihe des Instituts für Angewandte Informatik, (European Business School, 1992).

[14] B. L. Chalmers and G. D. Taylor, "Uniform approximation with constraints", Jahresber. Deutsch. Math.-Verein. 81 (1979) 49-86.

[15] R. A. DeVore, The approximation of continuous functions by positive linear operators (Springer, Berlin-Heidelberg-New York, 1972).

[16] R. A. DeVore and Xiang-ming Yu, "Pointwise estimates for monotone polynomial approximation", Constr. Approx. 1 (4) (1985) 323-331.

[17] G. Farin, Curves and surfaces for computer aided geometric design, 2nd ed. (Acad. Press, New York, 1990). 
[18] H. H. Gonska, "On Pičugov-Lehnhoff operators", Volume SM-DU-86 of Schriftenreihe des Fachbereichs Mathematik, (Universität Duisburg, 1985).

[19] H. H. Gonska, "Modified Pičugov-Lehnhoff operators", in Approximation theory V (Proc. Int. Sympos., College Station 1986) (eds. C. K. Chui et al.), (Acad. Press, New York, 1986) 355-358.

[20] J. Hoschek and D. Lasser, Grundlagen der geometrischen Datenverarbeitung, 2nd ed. (Teubner, Stuttgart, 1992).

[21] E. Isaacson and H. B. Keller, Analysis of numerical methods (John Wiley and Sons, New York, 1966).

[22] D. Leviatan, "Pointwise estimates for convex polynomial approximation", Proc. Amer. Math. Soc. 98 (1986) 471-474.

[23] G. G. Lorentz, "Inequalities and the saturation classes of Bemstein polynomials", in On Approximation Theory (Proc. Conf. Oberwolfach 1963) (eds. P. L. Butzer et al.), (Birkhäuser, Basel, 1964) 200-207.

[24] G. G. Lorentz, K. Jetter and S. D. Riemenschneider, Birkhoff interpolation (Addison-Wesley, Reading/ MA, 1983).

[25] G. G. Lorentz and K. L. Zeller, "Degree of approximation by monotone polynomials I", J. Approx. Theory 1 (1968) 501-504.

[26] Y. Matsuoka, "On the approximation of functions by some singular integrals", Tôhoku Math. J. 18 (1966) 13-43.

[27] R. Păltănea, "On the estimate of the pointwise approximation of functions by linear positive functionals", Volume 35 of Studia Univ. Babes-Bolyai Math., (1990) 11-24.

[28] J. Roulier, "Linear operators invariant on the nonnegative monotone functions", SIAM J. Numer. Anal. 8 (1971) 30-35.

[29] R. Senderovizh, "On convexity preserving operators", SIAM J. Math. Anal. 9 (1978) 157-159.

[30] A. S. Shvedov, "Jackson's theorem in $L^{p}, 0<p<1$, for algebraic polynomials and orders of comonotone approximations (Russian)", Mat. Zametki 25 (1) (1979) 107-117, Engl. transl.: Math. Notes 25 (1979), 57-63.

[31] A. S. Shvedov, "Orders of coapproximation of functions by algebraic polynomials (Russian)", Mat. Zametki 29 (1) (1981) 117-130, Engl. transl.: Math. Notes 29 (1981), 63-70.

[32] Xiang-ming Yu, "Pointwise estimates for convex polynomial approximation", Approx. Theory Appl. 1 (4) (1985) 65-74.

[33] A. Zygmund, Trigonometric series (Cambridge Univ. Press, Cambridge, 1959). 